Revista Calidad en la Educación Superior

Programa de Autoevaluación Académica

Universidad Estatal a Distancia, Costa Rica

ISSN 1659-4703

revistacalidad@uned.ac.cr

\title{
UNA EXPERIENCIA INTERUNIVERSITARIA Y MULTIDISCIPLINARIA DE AUTOEVALUACIÓN PARA LA ACREDITACIÓN: DOCTORADO EN CIENCIAS NATURALES PARA EL DESARROLLO
}

INTERUNIVERSITY AND MULTIDISCIPLINARY SELF-ASSESSMENT FOR ACCREDITATION: PHD IN NATURAL SCIENCES FOR DEVELOPMENT

\author{
Claudia Charpentier-Esquivel ${ }^{1}$ ccharpen@gmail.com \\ Instituto Tecnológico de Costa Rica \\ Carlos Hernández-Salazar ${ }^{2}$ carlos.hernandez.salazar@una.cr \\ Universidad Nacional de Costa Rica \\ Andrei Fedorov-Fedorov ${ }^{3}$ afedorov@itcr.ac.cr \\ Instituto Tecnológico de Costa Rica \\ Marlon Rojas-Sandí mrojass@uned.ac.cr \\ Universidad Estatal a Distancia, Costa Rica
}

\author{
Volumen 7, Número 2 \\ Noviembre 2016
}

pp. 1 - 24

Recibido: 30 de mayo, 2016

Aprobado: 29 de octubre, 2016

\footnotetext{
1 Dra. Claudia Charpentier-Esquivel del Tecnológico de Costa Rica (Coordinadora de la CAT). Correo electrónico: ccharpen@gmail.com

${ }^{2}$ MSc. Carlos Hernández-Salazar del Programa de Diseño y Gestión Curricular (PDGC, UNA). Correo electrónico: carlos.hernandez.salazar@una.cr

${ }^{3}$ Dr. Andrei Fedorov-Fedorov del Centro de Evaluación Académica (CEDA, TEC). Correo electrónico: afedorov@itcr.ac.cr

4 Lic. Marlon Rojas-Sandí del Instituto de Gestión de la Calidad Académica (IGESCA, UNED). Correo electrónico:

mrojass@uned.ac.cr
} 
Una experiencia interuniversitaria y multidisciplinaria de autoevaluación para la acreditación: Doctorado en Ciencias Naturales

Claudia Charpentier-Esquivel, Carlos Hernández-Salazar, Andrei Fedorov-Fedorov, Marlon Rojas-Sandí

\section{Resumen}

Desde la génesis del Programa de Doctorado en Ciencias Naturales para el Desarrollo (DOCINADE) en el 2003, se decidió que la evaluación sería parte de la agenda de trabajo y uno de los mecanismos regulares para el mejoramiento continuo. Con esa visión y aprovechando el camino transitado en el 2012 se formaliza la iniciativa de una autoevaluación con miras a la acreditación, por parte de Agencia Centroamericana de Acreditación de Posgrados (ACAP). Este esfuerzo se concreta en la presentación del respectivo informe en el año 2014. El proceso de autoevaluación reviste características especiales, que motivan la presente publicación, correspondiente a la naturaleza y particularidades de este Programa. Se trata de un doctorado interuniversitario, multidisciplinar, intercultural, de carácter Regional (América Latina) desarrollado de manera bimodal con un predominio de las actividades virtuales. Se ofrece desde Costa Rica, con la participación de tres de sus universidades estatales: la Universidad Nacional, el Instituto Tecnológico de Costa Rica y la Universidad Estatal a Distancia. La autoevaluación generó experiencias novedosas que se desean resaltar en este artículo, pues fueron factores importantes para una acreditación exitosa. Entre ellas destacan la definición de la estrategia metodológica para organizar y realizar la autoevaluación, la toma de decisiones por consenso entre las tres universidades, la armonía y respecto por los aportes de las diferentes formas de trabajo y sus contribuciones. La armonización de la participación de estudiantes, graduados y personal, la importancia del acompañamiento y de la asesoría metodológica y técnica de las oficinas responsables de la autoevaluación-acreditación en las tres instituciones estatales involucradas, fueron fundamentales en esta experiencia, que tiene como elemento integrador el objetivo de la calidad, la búsqueda de la excelencia y la certificación de ésta mediante su acreditación. Como resultado de este trabajo interuniversitario y multidisciplinario, se obtuvo un excelente informe final de autoevaluación, que permitió identificar las fortalezas del Doctorado y, por supuesto, aquellas áreas de oportunidad de mejora que se trasladaron a un plan de mejoramiento, que, a su vez, permitirá potenciar las fortalezas y superar esas debilidades detectadas. Se corona este esfuerzo con la acreditación otorgada por la ACAP y la entrega del respectivo certificado en mayo del 2016.

Palabras clave: autoevaluación, acreditación, calidad, Doctorado en Ciencias Naturales para el Desarrollo (DOCINADE), interuniversitario.

\section{Abstract}

Since the genesis of the PhD Program in Natural Sciences for Development (DOCINADE) in 2003, a decision was made, that evaluation would be part of the work agenda and one of the regular mechanisms for continuous improvement. With that vision and taking advantage of the path taken in 2012, the initiative of a self-assessment is formalized with a view to accreditation by Central American Accreditation Agency for Graduate Studies (ACAP). The effort concluded with the presentation of the respective report in 2014 . The self-assessment process had special characteristics that motivate this publication, 
corresponding to the nature and characteristics of this program. It is a doctorate, interuniversity, multidisciplinary, intercultural, regional (Latin America) developed bimodal with a predominance of virtual activities. Offered from Costa Rica with the participation of three of its state universities: the National University, the Costa Rica Institute of Technology and the State Open University. Self-assessment shows that novel experiences generated in the process of evaluation of this graduate program with accreditation purposes, which are emphasized in this article because they were important for the successful accreditation. These include the definition of the methodological strategy to organize and conduct self-evaluation, decision making by consensus between the three universities, harmony and respect for the contributions of the different forms of work and their contributions. The harmonization of the participation of students, graduates, faculty and staff and the importance of accompaniment and methodological and technical advice from the offices responsible for the self-accreditation in the three state institutions involved, were fundamental in this experience having the goal of quality, the pursuit of excellence, and this certification through its accreditation. Because of this interuniversity and multidisciplinary work, an excellent final self-assessment report is presented, which identified the strengths of the Doctorate and obtained those areas of opportunity for enhancement and moving into an improvement plan, which will augment the strengths and overcome those weaknesses detected. This effort concluded with the accreditation granted by the ACAP and the delivery of the respective certificate in May 2016.

Keywords: self-assessment, accreditation, quality, Doctorate in Natural Sciences for Development (DOCINADE), interuniversity. 


\section{Introducción}

La acreditación de las carreras universitarias es cada día más importante, y las universidades estatales costarricenses han hecho serios esfuerzos por lograr ese reconocimiento. Rojas (2003) y Campos y González (2006) hacen un recuento para la Universidad Estatal a Distancia (UNED); Fëdorov (2005, 2007, 2009) y Rodríguez, Zúñiga y Arnáez (2008) para el Instituto Tecnológico de Costa Rica (TEC) y Cedeño y Hernández (2011) y Hernández, Quesada y Sánchez (2005a y 2005b) para la Universidad Nacional (UNA).

La autoevaluación de carreras o programas universitarios con fines de acreditación y mejora continua, reviste un grado de complejidad reconocido, que permite lograr establecer las condiciones típicas de este tipo de proceso, como son: la planificación, recolección y sistematización de la información, el análisis, reflexión y valoración de la información, el aprendizaje, los planes de mejora, entre otros aspectos. Resulta, de por sí, laborioso en cualquier carrera o programa, y en muchos casos, estos procesos son innovadores (Hernández, Quesada, y Sánchez, 2005a y b.; Ugalde, 2008; Cox, 2010; González, Martínez, González y Hernández, 2012).

En el caso del Doctorado en Ciencias Naturales para el Desarrollo (DOCINADE), esta complejidad se incrementa en función de su naturaleza interuniversitaria, la fuerte proyección internacional y modalidad predominantemente virtual. Tomando en cuenta estas tres características distintivas del DOCINADE, se consideró relevante compartir el trabajo que se generó en busca de la acreditación de un programa doctoral bimodal, que puede servir como ejemplo del trabajo interuniversitario en su conceptualización, ejecución y evaluación.

Para una mejor contextualización y comprensión de la labor realizada, a continuación, se ofrece información general del doctorado y se comenta sobre la génesis del programa, las razones para acreditarse y la estructura organizativa del DOCINADE. También se 
explica la metodología que se empleó en el proceso de autoevaluación y se comenta acerca de la conformación, el rol y actividades desarrolladas por la Comisión de Asesoría Técnica (CAT), la cual resultó ser uno de los entes más importantes en la gestión de la autoevaluación.

\subsection{Génesis del DOCINADE}

El Doctorado en Ciencias Naturales para el Desarrollo es un programa interuniversitario que desarrollan tres universidades estatales costarricenses: el Instituto Tecnológico de Costa Rica (TEC), la Universidad Nacional (UNA) y la Universidad Estatal a Distancia (UNED). En el TEC están involucradas seis escuelas: la de Ingeniería Forestal, de Química, Biología, Ingeniería Electrónica, de Agronomía y de Ciencias Naturales y Exactas. En la UNA participan la Escuela de Ciencias Agrarias y la Escuela de Ciencias Ambientales de la Facultad de Ciencias de la Tierra y del Mar y la Escuela de Ciencias Biológicas de la Facultad de Ciencias Exactas y Naturales. Por su lado, en la UNED participan el Sistema de Estudios de Postgrado, el Centro de Capacitación a Distancia, la Escuela de Ciencias Exactas y Naturales, la Dirección de Tecnología de Información y Comunicaciones y la Dirección de Producción de Materiales Didácticos.

Además de las tres universidades costarricenses, participan dos universidades mexicanas en calidad de socias: la Universidad Nacional Autónoma de México - UNAM y la Universidad Autónoma de Chapingo - UACh, una universidad nicaragüense: la Universidad Autónoma de Nicaragua en León - UNAN y una universidad cubana, la Universidad de Ciego de Ávila - UNICA (Victorino, 2012).

Este programa nace como resultado de la discusión que efectuaron, inicialmente, profesores de las tres universidades costarricenses mencionadas. Ese grupo, multidisciplinario e interinstitucional decidió integrar a otros profesores de universidades latinoamericanas y europeas en el proceso de análisis. Su trabajo se basó en la 
elaboración de propuestas que permitieran la gestión de apoyo económico e integrar a otros docentes en la discusión.

De tal manera se logró que la Asociación Nacional de Universidades e Instituciones de Educación Superior (ANUIES) y el Consejo Superior de Universidades de Centro América (CSUCA) brindaran financiamiento para el desarrollo de cuatro convocatorias, entre los años 2001 y 2004. Ese apoyo permitió la incorporación de docentes de las universidades nacionales (TEC, UNA, UNED) e internacionales (UNAN-León, UNAM, UACh) en la discusión de la fundamentación, los perfiles y la propuesta final del plan de estudios (DOCINADE, 2014a; Victorino, 2012).

Por su parte, el Ministerio de Ciencia y Tecnología (MICIT) de Costa Rica brindó financiamiento del 2002 al 2009 para el transporte y manutención de académicos de México, Nicaragua, Cuba y España. Este apoyo económico ha permitido organizar seminarios y talleres internacionales en Costa Rica, Nicaragua y México desde el año 2005 hasta el 2009, donde se trabajó aspectos finales de la propuesta y de la implementación de las primeras generaciones (DOCINADE, 2014a).

Cabe mencionar que al igual que todo programa de posgrado, la propuesta de este doctorado debió pasar por cada instancia institucional de aprobación, solo que en este caso en las tres universidades nacionales, y finalmente se presentó al Consejo Nacional de Rectores (CONARE) para su aprobación. Inicialmente se aprobó con tres énfasis: Sistemas de Producción Agrícola (SPA), Gestión y Cultura Ambiental (GCA) y Gestión de Recursos Naturales (GRN). Además, en el 2008, se aprobó un cuatro énfasis: Tecnologías Electrónicas Aplicadas (TEA).

El programa fue aprobado por el Consejo Institucional del Instituto Tecnológico de Costa Rica y, posteriormente, también recibió la aprobación de los sistemas de estudio de posgrado de la UNA y de la UNED. Fue aprobado por el Consejo Nacional de Rectores (CONARE) en el 2003 y el 14 de febrero de 2005 los señores rectores firmaron los 
Una experiencia interuniversitaria y multidisciplinaria de autoevaluación para la acreditación: Doctorado en Ciencias Naturales para el Desarrollo

Claudia Charpentier-Esquivel, Carlos Hernández-Salazar, Andrei Fedorov-Fedorov, Marlon Rojas-Sandí

lineamientos para su puesta en marcha (DOCINADE, 2014a y b); la primera cohorte inició ese año.

\subsection{Estructura organizativa del DOCINADE}

EI DOCINADE dispone de una estructura organizativa en correspondencia con su génesis y lineamientos establecidos para su funcionamiento, que se muestra en la figura siguiente.

Figura 1. Organigrama del DOCINADE con sus comisiones y relaciones con las universidades

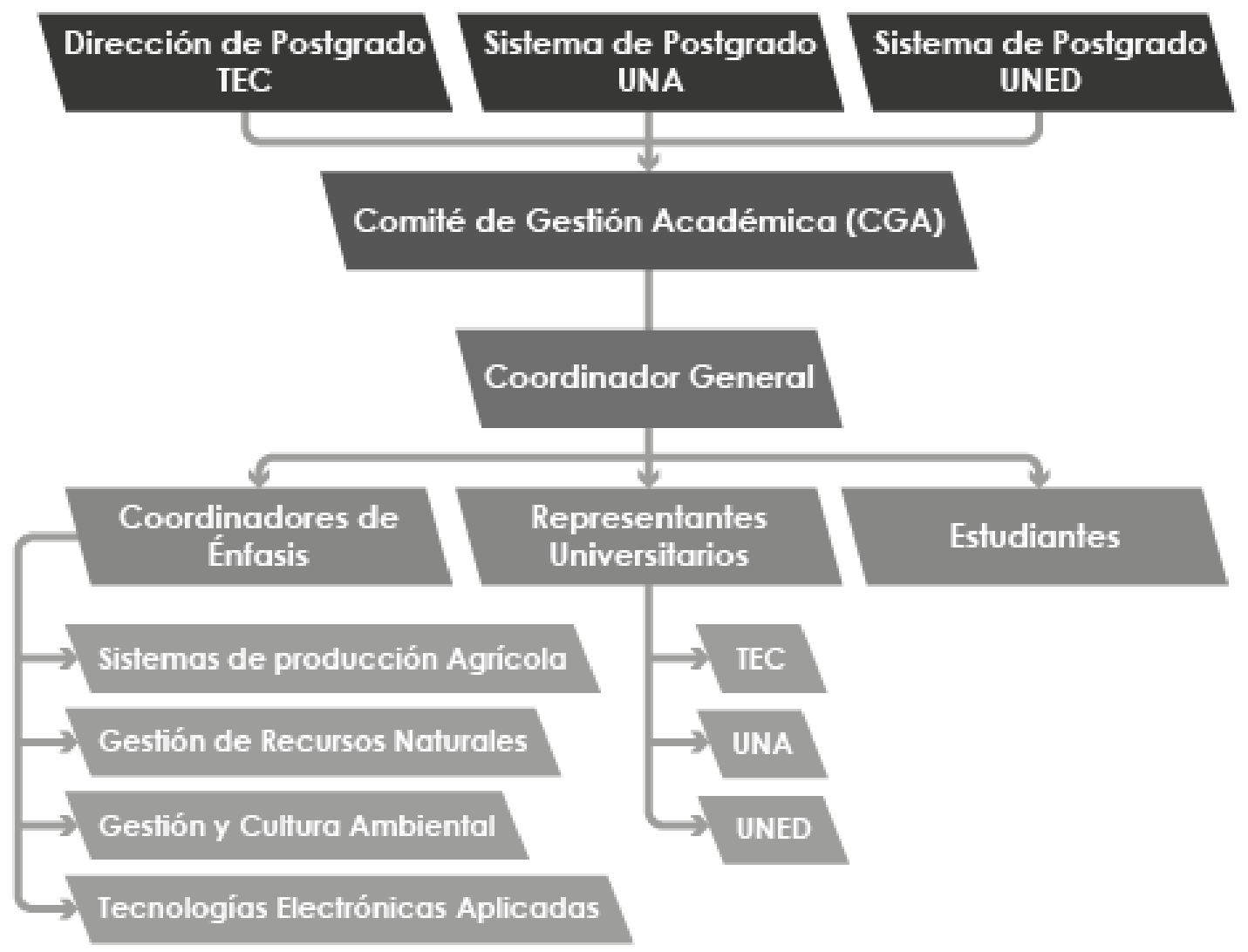

Fuente: Elaboró Andrei Fedorov 
El Comité de Gestión Académica (CGA) es el órgano responsable de la toma de decisiones en el doctorado. Está conformado por los representantes institucionales, los coordinadores de énfasis y los representantes estudiantiles. Cada institución tiene un representante designado por la Rectoría y un suplente. Los representantes postulan a los integrantes de las comisiones de énfasis y ellos a su vez designan al coordinador y al suplente. El Coordinador General es electo en el CGA y los postulantes son de ese cuerpo colegiado. Por su parte, los estudiantes eligen a quienes los representan en el CGA. Todos ellos forman parte del CGA.

La administración del doctorado es itinerante, el CGA aprueba la universidad que se encargará de la nueva cohorte de ingreso, también conocida como generación. Del 2005 al 2016 se tienen 8 cohortes, 4 son administradas por el TEC, 2 por la UNA y 2 por la UNED. Sus tareas para la atención del estudiantado, incluyen la realización de la matrícula, los trámites administrativos, el reporte de notas al departamento de registro, el seguimiento académico, la actualización de las bases de datos y la comunicación con el estudiante.

La universidad que administra debe contratar a los docentes para los cursos de la generación y la compra de bienes y servicios. También, participa en la toma de actas de las reuniones del Comité de Gestión Académica y esta tarea es rotativa para que el trabajo sea lo más equitativo posible.

A mayo del 2016, se han graduado 45 doctores y hay 3 egresados que estarían realizando las pre-defensas a junio del 2016. Esta es la dinámica actual del programa, todos los semestres hay pre-defensas y defensas de tesis. Los docentes que han participado en el doctorado provienen en su mayoría de Costa Rica, pero también de Nicaragua y México. Los tutores de tesis proceden de esos países, Colombia, Estados Unidos y España. 


\subsection{La autoevaluación: Motivos para realizarla}

Es reconocida la importancia de la autoevaluación de cualquier carrera o programa docente como mecanismo de mejora permanente de su calidad. Además, teniendo la aspiración de ostentar la condición de programa acreditado la autoevaluación es la etapa inicial imprescindible para llegar a esta certificación de la calidad del programa formativo (Cox, 2010; Hernández, Quesada y Sánchez (2005) y Fëdorov (2005).

En este programa se reconocieron características y necesidades específicas que justifican este proceso de autoevaluación: primeramente, la madurez académica alcanzada y, por tanto, la necesidad de evaluar el cumplimiento de los objetivos con los que fue creado.

Otra razón fundamental fue la necesidad de disponer del informe de autoevaluación que incluyera los logros obtenidos y el plan de mejoramiento, para compartir con las comunidades universitarias, egresados y graduados, así como con otros grupos de la sociedad, los cuales son fundamentales para el apoyo y sostenibilidad del doctorado. Esta primera autoevaluación con la participación de todas las cohortes busca ser el inicio de una cultura permanente de evaluación que debe caracterizar al DOCINADE, como apoyo a los procesos de mejoramiento continuo de un programa interuniversitario de calidad como éste.

Por otra parte, en este programa la administración de las cohortes es rotativa, de manera que todas las universidades puedan participar en la formación académica y además reciban y administren los ingresos producto del pago de matrícula. De esta manera se involucra a los departamentos financieros y de registro de todas las universidades participantes. Esta responsabilidad administrativa compartida es uno de los elementos de éxito del programa, porque todas las instituciones tienen las mismas tareas académicas y administrativas, pero deben identificar sus fortalezas y debilidades y participar en el plan de mejoramiento para mejorar los aspectos que así lo requieran. 


\section{Aportes a la autoevaluación: Metodología del proceso}

\subsection{Metodología empleada en la autoevaluación}

La decisión de realizar la autoevaluación, con fines de acreditación, siguiendo el modelo de la Agencia Centroamericana de Acreditación de Posgrados (ACAP), fue tomada de forma participativa y respondió, en parte, a la flexibilidad de la agencia acreditaria de permitir el ajuste de los indicadores a un programa interinstitucional y bimodal.

En la toma de esta decisión participaron: CGA, Direcciones de Posgrado, profesores del doctorado y el Vicerrector de Docencia del TEC. En este acuerdo incidió el hecho de que un número considerable de estudiantes son extranjeros, principalmente de la región mesoamericana, por lo cual la acreditación con una agencia de carácter regional se ajusta de mejor manera a los propósitos perseguidos.

En el 2012, se emprendieron las primeras acciones para evaluar la calidad del programa. En estas acciones se involucró el personal académico de las instituciones nacionales y extranjeras, al personal administrativo, los estudiantes y graduados. El proceso permitió identificar las fortalezas y las debilidades. Estas últimas se vieron como oportunidades para la mejora académica del programa y se incluyeron en el plan de mejoramiento.

En correspondencia con la naturaleza de este tipo de procesos, el de DOCINADE fue participativo en todas sus fases y promovió tanto la reflexión interna como la autocrítica, lo que permitió la identificación de elementos para la propuesta del plan de mejoramiento y de algunos indicadores fundamentales para el monitoreo y evaluación permanente del programa. Por esas razones, se buscó fortalecer las interrelaciones entre los académicos que conforman el programa, y entre ellos y los estudiantes y graduados. La dinámica principal de trabajo fue la conformación de comisiones, la consulta electrónica, la organización de reuniones y talleres con el fin de informar, analizar y evaluar los alcances del proceso en forma integral, considerando los aspectos que aportaron todas las 
personas involucradas. Posteriormente se detallará con más claridad las actividades que se realizaron como parte del proceso.

Para llevar a cabo esta tarea, la decisión del Comité de Gestión Académica (CGA) se comunicó a las instancias universitarias respectivas y a las vicerrectorías académicas y de docencia, las cuales brindaron el apoyo a través del Centro de Desarrollo Académico (CEDA) del TEC, del Programa de Diseño y Gestión Curricular (PDGC) de la UNA, y del Programa de Autoevaluación Académica (PAA) de la UNED. Las personas que se asignaron conformaron la Comisión de Asesoría Técnica (CAT) que acompañó este esfuerzo con miras a la acreditación ante ACAP.

Por tratarse de un programa interuniversitario, el papel de la Comisión de Asesoría Técnica fue sumamente importante, con aportes significativos de acuerdo con la forma en que cada universidad se organiza y realizan estos procesos para las carreras propias, rescatando y reconstruyendo la mejor ruta a seguir dada las características singulares de este programa. De esta manera, en un marco de respeto hacia el diálogo de saberes, el equipo llegó a consolidarse y generar un modelo de trabajo que es ejemplo de lo que se puede lograr en programas interinstitucionales. Este aspecto se retomará en este artículo más adelante, por ser uno de los elementos a destacar del proceso.

La figura 2 muestra gráficamente la forma en que se organizó la autoevaluación, considerando a todos los diferentes actores y su nivel de responsabilidad. 
Una experiencia interuniversitaria y multidisciplinaria de autoevaluación para la acreditación: Doctorado en Ciencias Naturales para el Desarrollo

Claudia Charpentier-Esquivel, Carlos Hernández-Salazar, Andrei Fedorov-Fedorov, Marlon Rojas-Sandí

Figura 2. Organización que se siguió para la autoevaluación.

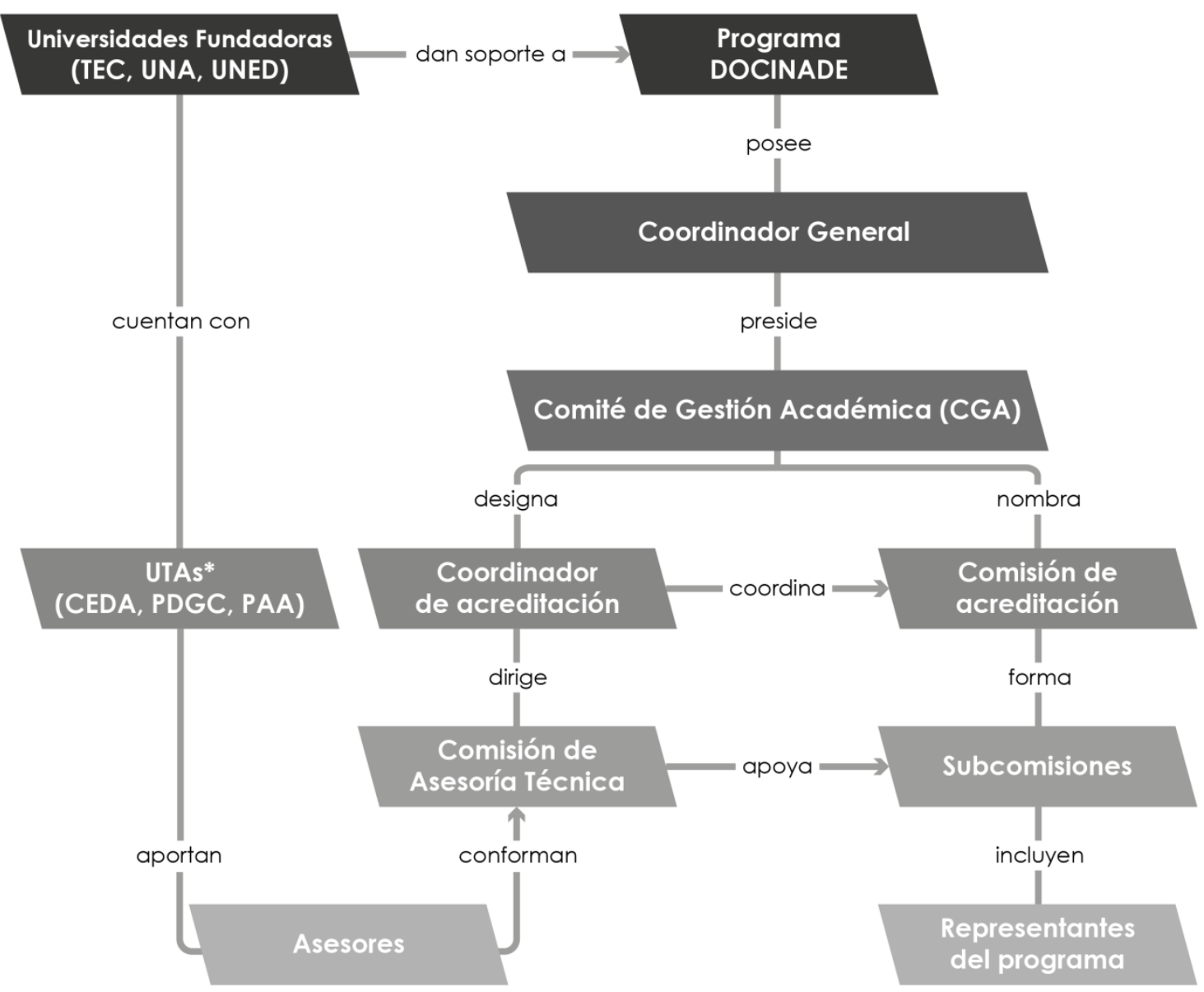

Nota: UTAs - unidades técnicas de apoyo a los procesos de autoevaluación y acreditación.

Fuente: Elaboró Andrei Fedorov

La organización que se logró para el proceso de autoevaluación fue determinante para el éxito. Se integraron los grupos necesarios para que el trabajo incluyera a la mayor cantidad de integrantes del CGA, personal docente, administrativo, estudiantes e integrantes de la Comisión de Asesoría Técnica. Como resultado, las tareas necesarias fueron responsabilidad de muchos, y la socialización de resultados y toma de decisiones 
Una experiencia interuniversitaria y multidisciplinaria de autoevaluación para la acreditación: Doctorado en Ciencias Naturales para el Desarrollo

Claudia Charpentier-Esquivel, Carlos Hernández-Salazar, Andrei Fedorov-Fedorov, Marlon Rojas-Sandí

fue más expedita. Todas las decisiones y propuestas fueron elevadas al CGA para su aprobación.

La figura 3, presenta el esquema general de la estrategia metodológica que se siguió en el proceso de autoevaluación. Se presenta las comisiones que trabajaron, la relación con el CGA y las otras instancias universitarias que participaron durante todo el proceso.

Figura 3. DOCINADE. Metodología empleada en la autoevaluación.

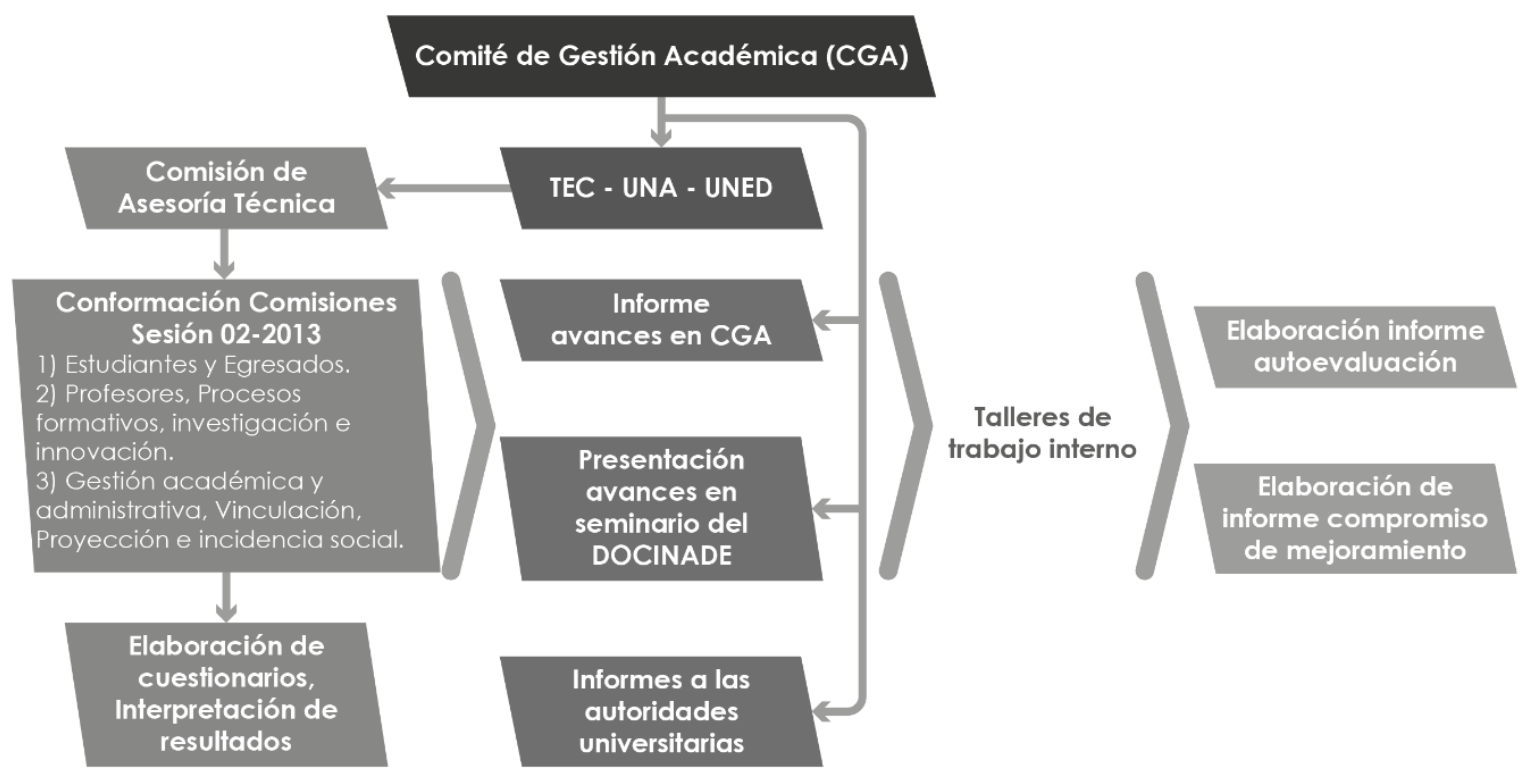

Fuente: Elaboró Andrei Fedorov

La autoevaluación incluyó cuadro fases: sensibilización, preparación, ejecución y síntesis evaluativa y se explican a continuación. 


\subsubsection{Fase de sensibilización}

Esta fase fue decisoria para lograr la alta participación de todos los grupos en el proceso, en la consolidación de las comisiones interuniversitarias de trabajo y para poner la casa en orden. El Comité de Gestión académica (CGA) la empezó durante los cursos y las actividades presenciales del DOCINADE que se realizan al inicio del año lectivo, donde informó que estaba empezando el proceso de acreditación e invitó a las personas a participar. Seguidamente, procedió a comunicar la decisión a las universidades y solicitó el nombramiento de los integrantes de la Comisión de Asesoría Técnica (CAT).

En esta fase fue fundamental para el trabajo coordinado entre las tres universidades, la participación de las asistentes administrativas y la construcción de una base de datos en Google Drive con los documentos generales del doctorado y los correspondientes a sus cohortes, que administró y gestionó la secretaria del TEC. Por su parte, las otras instituciones aportaron lo correspondiente a las cohortes suyas y se encargaron de mantener al día la información de las cohortes que administran. Esa base de datos se denominó "Ordenando la casa" y se comunicó los nombres de las personas que podrían tener acceso a esos documentos para que hiciera las autorizaciones respectivas. También, se acordó que la secretaría de la UNED asistiera a la Comisión de Asesoría Técnica y asumiera la elaboración de los archivos con los borradores de los anexos, algunos cuadros y figuras.

\subsubsection{Fase de preparación}

En esta fase se nombraron las tres comisiones de trabajo con representación de docentes, administrativas y estudiantes. Se elaboraron los cuestionarios dirigidos a docentes, estudiantes, graduados, administración y asistentes administrativas (DOCINADE, 2014aํ). Estos instrumentos fueron los que se estimó adecuados para un programa de doctorado con cuatro especialidades y que se desarrolla fundamentalmente a distancia. Los grupos de trabajo fueron la Comisión de Estudiantes y Graduados; la 
Comisión de Profesores, Proceso Formativo, Investigación e Innovación Comisión de Gestión Académica y la Administrativa, Vinculación, Proyección e Incidencia Social y Colaboración e Innovación. Las comisiones contaron con docentes de las tres universidades y el acompañamiento de uno de los integrantes de la Comisión de Asesoría Técnica. Es importante señalar que la segunda comisión fue coordinada por uno de los representantes estudiantiles del CGA y su trabajo tesonero sirvió como incentivo a las otras.

El trabajo de las comisiones fue fundamental para:

1. Elaborar los cuestionarios necesarios para recabar la información relativa a los indicadores de ACAP y a las particularidades del doctorado.

2. Validar los cuestionarios y remitirlos a la Comisión de Asesoría Técnica para su revisión y aplicación.

3. Interpretar los resultados de las encuestas para incluirlas en el informe de la comisión y remitirlo a la Comisión de Asesoría Técnica.

4. Brindar insumos para la propuesta del plan de mejoramiento del programa.

5. Aportar resultados que puedan considerarse en el proceso de toma de decisiones.

Por su parte, la Comisión de Asesoría Técnica revisó los cuestionarios y los envió al Programa de Autoevaluación Académica (PAA) de la UNED, para ponerlos en formato Lime Survey. Posteriormente, los cuestionarios se enviaron a estudiantes y docentes y los contestaron en línea. Es importante destacar el aporte del PAA, el cual aplicó y procesó la información para su análisis mediante la herramienta Lime Survey y la remitió a la coordinadora del proceso de autoevaluación. La Comisión de Asesoría Técnica coordinó el trabajo con la UNED, efectuó la primera revisión del informe que produjo la UNED y envió a las comisiones las partes que le correspondieron.

El proceso de encuesta y validación se hizo con el apoyo de las tecnologías de la información y comunicación, dado que el profesorado y estudiantado se encontraban en 
diferentes regiones del país y en diferentes países. Todas las sugerencias que brindaron fueron incorporadas para mejorar los cuestionarios.

\subsubsection{Fase de ejecución}

Esta fase incluyó los siguientes momentos:

- Aplicación de instrumentos en línea

- Procesamiento de la información y preparación de informes de resultados según grupo de encuestados

- Discusión de resultados preparados por las comisiones en el CGA

- Recolección de información documental

- Organización de la información

- Redacción del primer documento

El informe de las tres comisiones incluyó los resultados de opinión obtenidos y una breve discusión de los más relevantes. La recolección de información fue virtual, excepto en el caso de la comisión encargada de recabar información sobre Gestión Académica y Administrativa, Vinculación, Proyección e Incidencia Social y Colaboración e Innovación, que entrevistó al primer coordinador general y al actual. Los resultados de opinión de los encuestados se devolvieron a las comisiones para elaborar el informe de opinión y presentarlos al CGA y a la coordinadora del proceso de autoevaluación.

También, de forma virtual, se hizo la consulta para concretar la misión, visión y objetivos estratégicos del programa, sin embargo los resultados se compartieron en el CGA y ahí se aprobaron para incorporarlos en la base de datos y principales documentos del doctorado. 


\subsubsection{Fase de síntesis evaluativa}

Los informes de resultados de las encuestas de cada grupo que brindaron las comisiones y los documentos que se constituyeron en evidencias para cada uno de los indicadores (que se incluyen en los anexos del informe) se utilizaron para elaborar el informe de autoevaluación. Con el primer borrador del informe, de carácter general, fue posible construir la síntesis valorativa, de carácter cuantitativo, valorando aquellos aspectos que destacan como fortalezas y los aspectos a mejorar (debilidades).

Este primer borrador del informe de autoevaluación se remitió al Coordinador General y a la CAT para la revisión. La revisión minuciosa del CAT mejoró el informe, le dio una mejor integración y lo ajustó a las normas de ACAP. La nueva versión se remitió al Área Académica del Doctorado del TEC y, por último, al CGA para su estudio y aprobación final.

El CGA revisó las evaluaciones de cada uno de los componentes y posteriormente consideró los resultados para la elaboración del plan de mejora. En sesión de trabajo del CGA y del CAT se elaboraron las matrices del plan de mejoramiento, se jerarquizaron las acciones y se definieron las acciones del plan de mejoramiento que requieren apoyo institucional. Las sugerencias y recomendaciones que brindaron se incorporaron en la elaboración del informe de autoevaluación, plan de mejoramiento y resumen ejecutivo que se remitieron a las autoridades correspondientes de las universidades (vicerrectores y rectores).

Además, se hicieron presentaciones en el Instituto Tecnológico de Costa Rica, en la Universidad Nacional y la Universidad Estatal a Distancia. En la del TEC participaron autoridades y coordinadores de los programas de acreditación de las tres instituciones y los integrantes del equipo técnico. 


\subsection{Consolidación del Comité de Apoyo Técnico}

La consolidación del Comité de Apoyo Técnico y su trabajo es el aporte metodológico más importante del proceso. En Costa Rica, la acreditación del DOCINADE es el primer proceso que involucra a un programa de doctorado impartido por tres universidades nacionales estatales costarricenses con la colaboración de universidades extranjeras. Lo innovador de este proceso fue el compromiso de las autoridades de las instituciones y del doctorado y de los profesores para conciliar las particularidades de las tres y trabajar por el cumplimiento de tareas y metas trazadas para obtener la acreditación del DOCINADE.

Uno de esos compromisos fue el establecimiento de la CAT, con responsabilidades asignadas en el marco de sus jornadas laborales en cada universidad. Participaron el Dr. Andrei Fedorov Fedorov del Centro de Evaluación Académica (CEDA, TEC), MSc. Carlos Hernández Salazar del Programa de Diseño y Gestión Curricular (PDGC, UNA), Lic. Marlon Rojas Sandí del Programa de Autoevaluación Académica (PAA, UNED), la Dra. Claudia Charpentier Esquivel del Tecnológico de Costa Rica (Coordinadora de la CAT) y la Licda. Yeimy Jiménez Flores de la UNED, quien brindó el apoyo administrativo al grupo.

Los profesionales asignados a la CAT cuentan con gran experiencia en procesos de acreditación en las respectivas universidades, sin embargo, este proceso resultó novedoso para todos, pues participa más de una unidad académica en cada universidad y es un programa interinstitucional. Se integraron sus experiencias y saberes, pero sus principales logros fueron convertir potenciales barreras institucionales en oportunidades de trabajo conjunto y la manifestación del respeto a la autonomía y a los procesos propios de cada universidad. Situación que se pudo evidenciar en un ambiente de trabajo en armonía y en la ausencia de conflictos internos o externos. 


\subsubsection{Metodología de trabajo de la CAT}

La CAT generó una forma de trabajo que condujo al cumplimiento de las metas trazadas: el desarrollo de la autoevaluación y la elaboración del plan de mejoramiento del doctorado. Desde la primera reunión, se especificó la forma en que se trabajaría y las funciones y responsabilidades de los integrantes de esta comisión. Asimismo, cada uno de ellos definió con sus superiores la forma más expedita de trabajar y aprobar los informes y documentos que fueron presentados a las autoridades de las universidades y a ACAP, de manera que se aprobaran simultáneamente en las tres instituciones.

Cabe señalar que un aspecto requerido en este tipo de procesos es la definición de un plan de trabajo con responsabilidades claramente establecidas para todos los involucrados, y por supuesto, el cronograma de actividades, el cual en este caso, tuvo que ser ajustado en algunas oportunidades, debido al ritmo y cantidad de trabajo de cada grupo. No obstante, el plazo final que se tenía se cumplió.

La figura 4 muestra resumidamente la metodología que siguió la CAT para brindar el apoyo al proceso de autoevaluación del DOCINADE. Aunque en este esquema se muestra una secuencia lineal de acciones, es importante acotar que no siempre se realiza de tal manera, normalmente se da la realización de acciones de forma paralela en el tiempo. 
Figura 4. Metodología de trabajo de la Comisión Asesoría Técnica.

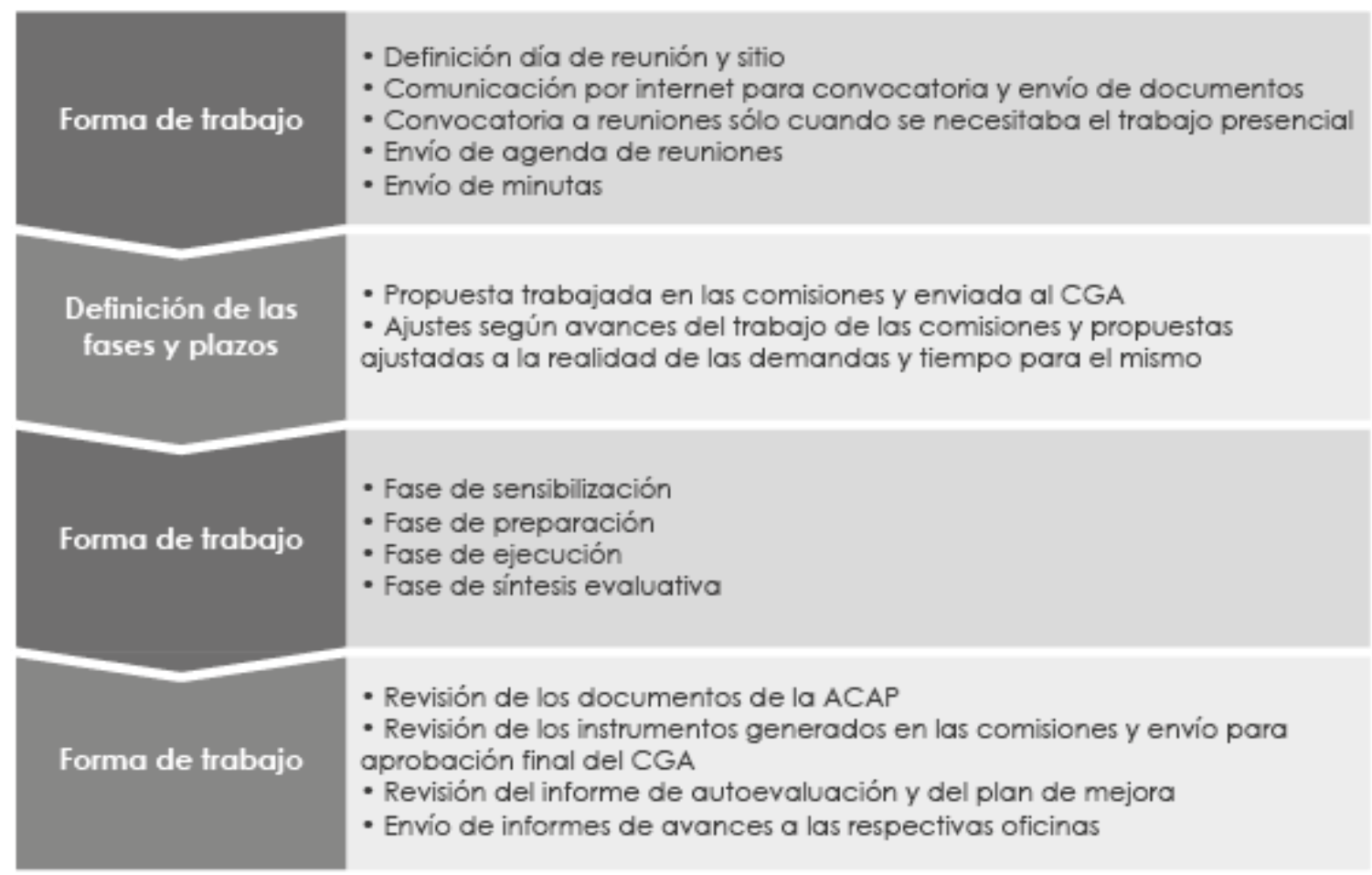

Fuente: Elaboró Andrei Fedorov

Los procesos de autoevaluación para lograr una acreditación de un programa suelen ser largos y desgastantes para quienes tienen la responsabilidad de coordinarlos y trabajarlos, porque por lo general se involucra a una pequeña comisión. No obstante, en el caso del DOCINADE, se contó con un factor diferenciador al lograr una organización que permitió la participación de un mayor número de personas, debidamente articuladas desde la función de CAT y su coordinación.

De tal manera, se trabajó no con una comisión, sino con tres comisiones (temáticas), entrelazadas y articuladas por los miembros de la CTA, y la persona que estuvo a cargo de la coordinación de esta última comisión fue la Dra. Claudia Charpentier, quien movió los hilos articuladores entre todas las partes y en todas las direcciones. Es decir, en el plano horizontal y en el plano vertical. Esta figura resulta de trascendental importancia 
en el tanto permite la comunicación entre las partes, y, en especial, hacia y desde los órganos directores del programa. Es digno de destacar, como enseñanza, la necesidad de contar en la coordinación funcional de este tipo de procesos con una persona que cumpla con al menos tres siguientes características: 1) conocedora del objeto que se evalúa (programa), 2) conocedora de la practica evaluativa, y 3) experiencia en investigación y, por supuesto, otras competencias como son el liderazgo y la comunicación.

En esa definición de responsabilidades, los integrantes de la CAT apoyaron en la clarificación de conceptos, brindaron información durante las etapas del proceso y en la revisión de informes y documentos que se utilizaron para consolidar el informe de autoevaluación y del plan de mejoramiento (DOCINADE, 2014). Su trabajo cordial y tesonero en las comisiones se convirtió en un elemento determinante para la participación de los profesores. A la vez pudieron vivenciar el trabajo del CGA y las diferentes reuniones que se concertaron para revisar y aprobar los avances y las versiones finales de los documentos que se presentaron a las autoridades de las universidades.

\section{Conclusiones}

La estrecha relación durante todo el proceso con los directores del Centro de Desarrollo Académico (CEDA) del TEC, del Programa de Diseño y Gestión Curricular (PDGC) de la UNA, y del Programa de Autoevaluación Académica (PAA) de la UNED, hizo muy expedita la aprobación del informe final y del plan de mejoramiento que se presentó para aprobación de las autoridades de las tres universidades y a la ACAP.

La participación de los integrantes de la CAT en las comisiones conformadas para el trabajo fue un acierto y parte medular del éxito que se tuvo en las diferentes fases del proceso. Siempre estuvieron en las sesiones e hicieron las sugerencias sobre la marcha 
Una experiencia interuniversitaria y multidisciplinaria de autoevaluación para la acreditación: Doctorado en Ciencias Naturales

Claudia Charpentier-Esquivel, Carlos Hernández-Salazar, Andrei Fedorov-Fedorov, Marlon Rojas-Sandí

del trabajo y no hasta el final, de manera que se hicieron las correcciones o adiciones y se avanzó mucho más rápido.

El apoyo del Coordinador General del DOCINADE y del CGA en el trabajo de las comisiones y en la atención a las solicitudes de la CAT, de forma pronta y con la dedicación de tiempo y recursos financieros necesarios, fueron acciones fundamentales y oportunas para cumplir con el cronograma y fases propuestos.

El proceso participativo que se siguió para la autoevaluación y el acompañamiento de la CAT fueron unos de los elementos que permitieron concluir en el tiempo programado las metas trazadas para lograr la acreditación del DOCINADE.

El apoyo de las autoridades universitarias, de los directores de las oficinas responsables de los procesos de acreditación y el compromiso de docentes, estudiantes, administrativos y de la CAT se convirtieron en el complemento necesario para el éxito del proceso.

\section{Referencias}

Campos, J. y González, N. (2006). Los procesos de autoevaluación desarrollados en la UNED durante el periodo 2002-2005: Análisis comparativo. Vicerrectoría Académica, Programa de Autoevaluación Académica. Recuperado de www.uned.ac.cr/academica/images/.../INVEST2.PDF. 53 p.

Cedeño, M.A. y Hernández, C. (2011). Autoevaluación y acreditación de carreras en la Universidad Nacional de Costa Rica: Experiencias para la gestión de la calidad. Revista Calidad en la Educación Superior, 2(2), 44-68. Recuperado de https://investiga.uned.ac.cr/revistas/index.php/revistacalidad

Cox, J. (2010). Autoevaluación y acreditación por agrupamientos (clústeres) en la Escuela de Ciencias Administración de la Universidad Estatal a Distancia de Costa Rica: un nuevo enfoque para el abordaje de la autoevaluación y acreditación en la educación superior. Revista Calidad en la Educación Superior, 1(2), 9-30. Recuperado de https://investiga.uned.ac.cr/revistas/index.php/revistacalidad 
Una experiencia interuniversitaria y multidisciplinaria de autoevaluación para la acreditación: Doctorado en Ciencias Naturales

Claudia Charpentier-Esquivel, Carlos Hernández-Salazar, Andrei Fedorov-Fedorov, Marlon Rojas-Sandí

Doctorado en Ciencias Naturales para el Desarrollo (DOCINADE). (2014a). Informe de autoevaluación para la Agencia Centroamericana de Acreditación de Posgrados $(A C A P)$.

Doctorado en Ciencias Naturales para el Desarrollo (DOCINADE). (2014b). Plan de mejoramiento para la Agencia Centroamericana de Acreditación de Posgrados $(A C A P)$.

Fëdorov, A. (2005). Nuevo panorama de los procesos de acreditación de las carreras de ingeniería y de arquitectura en Costa Rica. Innovación Educativa, 5 (28), 45-55.

Fëdorov, A. y Guzmán, I. (2007). Licenciatura en ingeniería agrícola del Instituto Tecnológico de Costa Rica: Desarrollo e innovación en el curriculum del programa. Innovación Educativa, 7(37), 5-15.

Fëdorov, A. (2009). Internacionalización de los planes de estudio de las ingenierías: Una aproximación instrumental. Innovación Educativa, 9(48), 5-17. Accesible en http://www.redalyc.org/pdf/1794/179414896002.pdf

González, E., Martínez, M., González, E. y Hernández, C. (2012). Acreditación y reacreditación en la Universidad Nacional (Costa Rica): dos experiencias de mejoramiento continuo. Revista Bibliotecas, 30(2), sp. Recuperado de www.revistas.una.ac.cr/index.php/bibliotecas.

Hernández, C., Quesada, E. y Sánchez, V. (2005a). Efectos de los procesos de autoevaluación de carreras en el mejoramiento de la gestión universitaria'. Simposio Internacional: de la evaluación acreditación al mejoramiento de la calidad de la educación superior. San José, Costa Rica.

Hernández, C., Quesada, E. y Sánchez, V. (2005b). Búsqueda de Condiciones que Garanticen Procesos Exitosos de Autoevaluación: Lecciones desde la autoevaluación de carreras en la Universidad Nacional. Simposio Internacional: de la evaluación acreditación al mejoramiento de la calidad de la educación superior. San José, Costa Rica

Rojas, R. (2003). El proceso de autoevaluación de programas académicos: aprendizajes de la experiencia en la UNED de Costa Rica. Revista Iberoamericana de Educación a Distancia, 6(2), 121-139. Recuperado de https://dialnet.unirioja.es/ejemplar/130982

Rodríguez, U., Zúñiga, C. y Arnáez, E. (2008). Factores que contribuyen con el éxito en los procesos de autoevaluación con miras a la acreditación. Experiencia del Instituto Tecnológico de Costa Rica. Actualidades Investigativas en Educación, 8(1), 1-19. Recuperado de http://revista.inie.ucr.ac.cr 
Una experiencia interuniversitaria y multidisciplinaria de autoevaluación para la acreditación: Doctorado en Ciencias Naturales para el Desarrollo

Claudia Charpentier-Esquivel, Carlos Hernández-Salazar, Andrei Fedorov-Fedorov, Marlon Rojas-Sandí

Ugalde, A. (2008). Acreditación de la Bibliotecología y Documentación en Costa Rica. Escuela de Bibliotecología, Documentación e Información, Universidad Nacional. Revista Bibliotecas, 26(2), sp. Recuperado de www.revistas.una.ac.cr/index.php/bibliotecas.

Victorino-Ramírez, L. (2012). Programa de postgrado interuniversitario doctorado en ciencias naturales para el desarrollo: antecedentes, actualidad y perspectivas como experiencia de gestión del conocimiento. Recuperado de dialnet.unirioja.es/descarga/articulo/4636815.pdf 